calculate 'free-living' energy expenditure in third trimester pregnant women in rural Nepal.

Methods Three-week time limited study. Sixteen third trimester rural women from Jumla district Nepal were selected to wear the Actiheart device for $24 \mathrm{~h}$. Participants were recruited by moving house to house in small village communities. All participants were agricultural labourers responsible for small marginal farms and household chores.

A Nepali nurse gathered informed verbal consent from the women, measured BMI and assessed their trimester by questioning.

The Actiheart device has been validated against the doubly labelled water method as the gold standard of energy expenditure measurement.

Participants were excluded from the study if the Actiheart device was worn for $<20 \mathrm{~h}$ or if heart rate trace was $<80 \%$ complete. Data was analysed using Actiheart software.

Results 16 participants were measured during the three-week period and five were excluded from the sample due to insufficient heart rate trace or time wearing the Actiheart.

Physical activity level (PAL) category boundaries stated by the WHO, showed 4/11 women had a 'light' PAL (1.40-1.69), 3/11 'moderate' PAL (1.70-1.99) and 4/11 'vigorous' activity level (>2).

The sample average PAL was 1.85 , significantly higher than that reported in studies of pregnant women in developed countries such as the UK (1.62) and Sweden (1.65).

$8 / 11$ participants were estimated to be in negative energy balance when using the total daily energy intake of $2140 \mathrm{kcal}$ reported in pregnant women in the nearby area of Tamang.

Conclusion Pregnant women in rural Nepal have heavy workloads and high physical activity levels in the third trimester. High maternal energy expenditure and poor nutrition may result in a negative energy balance leading to weight loss, low birth weight and poor maternal and neonatal outcomes.

\section{G262(P) PAEDIATRIC DISABILITY IN MALAWI: EXPLORING INFORMATION AND ADVICE NEEDS AT COMMUNITY LEVEL}

${ }^{1} \mathrm{O}$ Shanahan, ${ }^{2} \mathrm{M}$ Mallewa, ${ }^{3} \mathrm{M}$ Wazakili, ${ }^{2} \mathrm{~J}$ Chimoyo, ${ }^{4} \mathrm{M}$ Gladstone. ${ }^{1}$ Liverpool School of Tropical Medicine, Faculty of Health and Life Sciences, University of Liverpool, Liverpool, UK; ${ }^{2}$ Malawi Liverpool Wellcome Trust, Blantyre, Malawi; ${ }^{3}$ Ministry of Disability, Malawi; ${ }^{4}$ Department of Women's and Children's Health, Institute of Translational Medicine, University of Liverpool, Liverpool, UK

\subsection{6/archdischild-2015-308599.255}

Aims To assess the perceptions and experiences of families and community workers who manage children with disabilities in rural Malawi to enable the cultural adaptation of a training intervention. Disability information and advice services in this setting are limited despite the burden of paediatric disability on many families in Malawi. The study aims to clarify the needs and priorities of families and health-workers, existing practices and training, in order to inform the development of an efficacious training programme for community workers to improve support to families of children with disabilities.

Methods The study was conducted in Thyolo, a southern district of Malawi. Sampling was purposive and recruitment was via key informants, community health personnel and volunteers across four traditional areas. 17 interviews and 4 focus groups were completed with parents/carers of children with disabilities and 5 interviews were conducted with community workers, a total of 53 participants. Interviews and focus groups were conducted and recorded in Chichewa/English by a Malawian research assistant, then transcribed and translated. Analysis involved a thematic framework approach using NVIVO 10.

Results Parents discussed many complex issues. Their main challenges included discrimination in the community, a lack of information and general advice on disability, childcare management, the need for prognosis counselling and a lack of resources for transport, medications and SNE. On the whole, community workers felt that they needed more resources in order to provide outreach services. At present, they primarily act as disability awareness campaigners and provide referral services to other institutions. Access and transport were the main concerns raised, together with a lack of specific disability training.

Conclusion Lack of disability services, community-worker training, poor communication and discrimination of those with disabilities are significant barriers to the effective management of disabled children in rural Malawi. A programme providing specific disability training to community workers, sensitisation and education of communities on a local level, together with involvement of all relevant stakeholders, is necessary to overcome these factors and improve support to families of children with disabilities.

\section{G263(P) ABSTRACT WITHDRAWN}

\section{G264(P) CEREBROVASCULAR ACCIDENT AMONG CHILDREN WITH SICKLE CELL ANAEMIA IN MULAGO HOSPITAL, UGANDA}

${ }^{1} \mathrm{D}$ Munube, ${ }^{2} \mathrm{P}$ Lwabi, ${ }^{1} \mathrm{G}$ Ndeezi, ${ }^{1} \mathrm{JK}$ Tumwine. 'Department of Paediatrics and Child Health, School of Medicine, College of Health Sciences, Makerere, Kampala, Uganda; ¿Uganda Heart Institute, Mulago Hospital, Kampala, Uganda

\subsection{6/archdischild-2015-308599.256}

Background Sickle cell anaemia (SCA) is a leading cause of child morbidity and mortality affecting 4\% of all newborns in Uganda. Cerebrovascular accident (CVA) is a serious complication of SCA in childhood and $10 \%$ of patients with SCA will have had a CVA by the age of 20 years. Hitherto there has been limited information on SCA-related stroke in Uganda.

Objective To describe the clinical presentation of children with SCA-related stroke; and haematological indices and interventions in those with and without CVA.

Method We studied 184 SCA children aged 2-18 years attending the sickle cell services of Mulago Hospital in Uganda from February to June 2011. Forty six children with CVA were compared with 138 without CVA. Analysis was done using chi square and Fisher's exact test.

Results There were 184 SCA patients: 46 with CVA and 138 without CVA. The median age at first CVA was 4 years [Interquartile range (IQR) 2-6]. Symptoms of children with SCArelated stroke included behaviour changes, headache and seizures. Findings on physical examination included hemiplegia (37), aphasia (10), and limb ataxia (16). Four of the 46 children with SCA-related stroke had an $\mathrm{HbS}<30 \%$, versus $0 / 138$ without CVA $(p=0.004)$. Children with SCA-related stroke $(44 / 46)$ were more likely to have received a blood transfusion than those without CVA (99/138): OR 8.6 (95\% CI 2.0, 37.5).

Conclusion Cerebrovascular accident in SCA patients is a serious and devastating complication. Patients presented with several neurological signs and symptoms. Further studies to elucidate the outcome and management are urgently needed. 